\title{
Qualidade e diversidade institucional na pós-graduação brasileira
}

JOÃO E. STEINER

\section{Introdução}

$\mathrm{U}$ NIVERSIDADES DE PESQUiSA e de pós-graduação compõem o topo da pirâmide do sistema de ensino. A maior parte dos países tem um sistema diversificado e robusto, pois na era da sociedade do conhecimento, nenhum país pode dispensá-lo.

Dizem os especialistas que a diversidade biológica é base de riqueza - uma espécie de lei fundamental da evolução. Daí a preocupação planetária em preservar a sua biodiversidade. No caso de instituições de ensino, a diversidade institucional também seria fonte de riqueza? O exemplo dos EUA, que têm, talvez, o sistema mais diversificado e bem-sucedido do planeta, parece indicar que sim. Com diversidade e liberdade de organização, afloram as potencialidades individuais e institucionais. Na maior parte dos países desenvolvidos e em desenvolvimento, o ensino superior é composto de um sistema complexo e diverso. $\mathrm{Na}$ Europa, por exemplo, cada país tem um sistema próprio que varia de caso a caso, inclusive com um sistema complexo de diplomas. Nos Estados Unidos, por outro lado, existem as universidades públicas, as universidades privadas com ou sem fins lucrativos e os Colleges com cursos de duração diversificada, além de um sem número de instituições de natureza diversa. No Brasil, a diversidade institucional do sistema cresceu nos anos de 1990, passando a desempenhar uma pluralidade de funções na formação acadêmico-profissional (Martins, 2000). Do ponto de vista legal, o sistema está constituído de universidades, centros universitários, faculdades reunidas e faculdades (Decreto no 3.860 de 9/7/2001). Elas podem ser instituições públicas ou privadas e estas últimas podem ser comunitárias, confessionais, ou empresariais. Nem todas as universidades são universidades de pesquisa e as que o são, não fazem toda a pesquisa com a mesma intensidade (Lobo, 2004) assim como nem todas são de pós-graduação e as que são, não a fazem da mesma forma. A nomenclatura oficial não oferece formas objetivas e precisas de caracterização dessa diversidade. Pouco nos diz sobre a existência ou não de pós-graduação e pesquisa, ou sobre abrangência e tamanho. Daí a necessidade de uma forma mais precisa de caracterização institucional.

A avaliação, por outro lado, é parte essencial da atividade acadêmica. Não só a avaliação estudantil, mas também a docente, são práticas universais (ou quase). 
A avaliação institucional é menos comum, porém, em um sistema educacional robusto, ela é fundamental para dar transparência aos usuários e à sociedade. No caso da pós-graduação brasileira, a Capes (Coordenação de Aperfeiçoamento do Pessoal de Nível Superior, do Ministério da Educação) vem realizando um trabalho paradigmático de avaliação dos programas ao longo de muitos anos.

No presente trabalho, descrevemos as instituições de pesquisa e de pósgraduação utilizando os critérios adaptados da Carnegie Foundation, tais como definidos por Steiner (2005). Analisamos as principais características do sistema e fazemos algumas considerações sobre seus pontos fortes e fracos.

\section{Diversidade institucional}

Como caracterizar a diversidade institucional? A Carnegie Foundation formulou um sistema pelo qual todas as instituições de ensino superior nos Estados Unidos podem ser classificadas. A primeira classificação foi formulada em 1971 sob a coordenação do educador Clark Kerr e teve a primeira versão publicada em 1973, tendo sido atualizado em 1976, 1987, 1994 e 2000. Para o ano de 2005, está prevista uma nova versão. A China também possui um sistema de classificação de instituições de ensino superior (Liu). No Brasil o sistema de ensino superior cresceu e se diversificou muito nas últimas décadas mas, apesar disso, não existia, no nosso país, um sistema de classificação como o da Carnegie (Lobo, 2004).

Para a nossa realidade, o critério de classificar as instituições com base na pós-graduação é mais objetivo do que o embasado na pesquisa, uma vez que não existem critérios homogêneos que englobem a real produção de todas as áreas do conhecimento. No Brasil, as áreas de ciências da natureza têm sua produção publicada basicamente em língua inglesa e a área das humanidades, em língua portuguesa. Já a formação de mestres e doutores é uma métrica mais uniforme.

Com base na classificação Carnegie, Steiner (2005) propôs uma adaptação para o Brasil. A classificação proposta está baseada em três dimensões:

- O grau mais elevado de diplomação:

- doutorado;

- mestrado;

- graduação.

- A diversidade acadêmica e o tamanho:

- diversificadas;

- intermediárias;

- restritas;

- especializadas.

- A natureza administrativa:

- públicas (federais, estaduais e municipais);

- CCF (comunitárias, confessionais e filantrópicas); e

- particulares (de caráter empresarial, não comunitário).

As instituições de pós-graduação estão listadas no Apêndice, de acordo com as diversas categorias. 


\section{Algumas estatísticas}

As instituições brasileiras de doutorado e de mestrado, agrupadas de acordo com os critérios acima, estão listadas no apêndice. Com base nessa lista podemos realizar algumas estatísticas.

As universidades e institutos públicos são amplamente dominantes entre as instituições de doutorado. As instituições comunitárias/ filantrópicas têm uma participação significativa nas instituições de doutorado e crescente nas de mestrado e já são duas vezes mais freqüentes entre as instituições de graduação. Já as instituições particulares estão totalmente ausentes entre as instituições de doutorado e as de mestrado diversificadas. Elas já têm, no entanto, uma presença significativa e crescente entre as instituições de mestrado intermediárias, restritas e especializadas. Entre as instituições de graduação, elas são amplamente dominantes do ponto de vista numérico.

Tabela 1 - Número de instituições por tipologia

\begin{tabular}{l|c|c|c}
\hline & Pub & CCF & Part \\
\hline Categ. & $\mathrm{Nr}$ & $\mathrm{Nr}$ & $\mathrm{Nr}$ \\
\hline
\end{tabular}

\begin{tabular}{l|c|c|c}
\hline DrDiv & 9 & - & - \\
\hline Drlnt & 8 & 3 & - \\
\hline DrRes & 14 & 4 & - \\
\hline DrEsp & 5 & 2 & - \\
\hline
\end{tabular}

\begin{tabular}{l|c|c|c}
\hline MsDiv & 8 & 7 & - \\
\hline MsInt & 6 & 8 & 5 \\
\hline MsRes & 7 & 9 & 5 \\
\hline MsEsp & 11 & 3 & 5 \\
\hline
\end{tabular}

\begin{tabular}{l|c|c|c}
\hline GrDiv & 9 & 9 & 13 \\
\hline Grlnt & 11 & 29 & 31 \\
\hline GrRes+Esp & 110 & 247 & 1075 \\
\hline
\end{tabular}

As nove universidades de pesquisa e doutorado diversificadas são instituições de referência nacional ou regional. Todas elas são públicas, sendo três estaduais (paulistas) e seis federais. A distribuição regional é bastante irregular, sendo que cinco se localizam no Sudeste (USP, Unicamp, Unesp, UFRJ e UFMG), 
duas no Sul (UFRGS e UFSC), uma no Nordeste (UFPE), uma no Centro-Oeste $(\mathrm{UnB})$ e nenhuma na região Norte.

Quanto ao número de concluintes, as proporções variam muito no que diz respeito ao doutorado, ao mestrado e à graduação, como pode ser atestado na Tabela 2 .

Tabela 2 - Número de concluintes de doutorado, mestrado e graduação por categoria institucional

\begin{tabular}{l|c|c|c}
\hline CATEGORIA & \multicolumn{3}{|c}{ CONCLUINTES } \\
\hline & Dr & Ms & Gr \\
\hline DrDiv & 5.485 & 12.293 & 30.593 \\
\hline DrInt & 1.405 & 5.164 & 26.374 \\
\hline DrRes & 584 & 3.646 & 34.551 \\
\hline DrEsp & 448 & 1.017 & 373 \\
\hline
\end{tabular}

\begin{tabular}{l|c|c|c}
\hline MsDiv & 128 & 1.949 & 41.089 \\
\hline Msint & 35 & 1.363 & 48.873 \\
\hline MsRes & - & 657 & 32.866 \\
\hline MsEsp & 54 & 808 & 900 \\
\hline
\end{tabular}

\begin{tabular}{l|c|c|c}
\hline GrDiv & 9 & 101 & 74.135 \\
\hline Grint & 0 & 95 & 76.006 \\
\hline GrRes+Esp & - & - & 163.000 \\
\hline
\end{tabular}

No Brasil, foi recentemente instituído o mestrado profissionalizante, tendo graduado os primeiros alunos em 1999. O crescimento nesses primeiros anos tem sido muito significativo. Apesar de esta iniciativa ainda ser incipiente, já está claro que as universidades maiores e mais tradicionais não incorporaram esta nova modalidade de forma significativa. Nas universidades de pesquisa e de doutorado, apenas $4 \%$ dos mestrados foram profissionalizantes no ano de 2003 . Já entre as universidades de mestrado, esta fração foi de $9 \%$ e entre as instituições especializadas, de 28\%. As universidades públicas (com 5\%) e as comunitárias/ filantrópicas (com 6\%) também não investiram nesta modalidade, ao passo que nas instituições particulares 33\% dos mestrados obtidos em 2003 foram profissionalizantes. Fica claro, assim, que esta nova modalidade de mestrado profissionalizante tem oferecido oportunidades tanto para as instituições especializadas como para as particulares. 
A introdução do mestrado profissionalizante foi um passo que aumentou a diversidade de diplomas na pós-graduação, antes restrita aos títulos de doutorado e mestrado acadêmico. Trata-se, pois, de uma medida que aumentou a flexibilidade do sistema.

\section{Sobre a história e a geografia}

O ensino superior em geral e a fundação de universidades, em particular, tiveram início tardio no Brasil, tendo suas primeiras iniciativas muito depois dos EUA e de outros países da América do Sul. Os primeiros cursos superiores no Brasil instalaram-se no início do século XIX. Com a vinda de D. João VI ao Brasil em 1808, foram instalados os três primeiros cursos médico-cirúrgicos, um em Salvador e dois no Rio de Janeiro. Em 1810, foi fundada a Academia Real Militar (hoje Escola de Engenharia da UFRJ). As Faculdades de Direito de São Paulo (Largo São Francisco) e de Olinda, foram fundadas em 1827. No final do Segundo Império e no início da República, foram fundadas escolas profissionais como Medicina, Engenharia, Agricultura e Direito. A Escola de Minas de Ouro Preto (1875), a Escola Politécnica de São Paulo (1893), a Escola de Engenharia Mackenzie (1896), a Escola de Engenharia de Porto Alegre (1886), a Escola Livre de Farmácia de São Paulo (1898) e a Escola Superior de Agronomia e Veterinária do Rio de Janeiro (1898) são alguns exemplos.

No início do século XX, já na fase republicana, intensificou-se a criação de escolas profissionais e algumas delas começaram a se reunir para formar as primeiras universidades; a UFPR (1912), a PUC-RS (1924) e a UFMG (1927) foram as primeiras. Todas elas eram escolas que visavam à transmissão da cultura $\mathrm{e}$, principalmente, à formação para as profissões, não havendo o conceito de pesquisa ligada ao ensino, já comum nesta época nas universidades humboldtianas (que associavam ensino e pesquisa) da Europa e EUA. A política brasileira era dominada por oligarquias agrárias conservadoras, a chamada política do café com leite. A Revolução de 1930 levou Getúlio Vargas ao poder, quebrando essa estrutura. Foi nomeado, então, o primeiro Ministro da Educação e Saúde, Francisco Campos, que promoveu uma reforma conservadora do ensino superior, nos moldes ultrapassados das universidades pré-humboldtianas. As universidades que já existiam e as que se formaram a partir de então, passaram a ter este caráter, com exceção da USP, a Universidade de São Paulo, criada pelo governo estadual paulista.

Até o final da década de 1950, já existiam nove universidades federais, duas estaduais e oito confessionais. Na década de 1960, houve dois surtos de criação de universidades que hoje são de pesquisa, ambos em fases conturbadas da vida política brasileira (ver Figura 1). O primeiro nos anos de 1960-1962, final da presidência de Juscelino Kubitscheck, no curto período de Jânio Quadros e no primeiro ano da fase parlamentarista de João Goulart. Neste período também houve uma intensificação do movimento estudantil, vitaminado pela "crise dos excedentes" (Durham, 2005). O segundo surto ocorreu no contexto da reforma 
universitária de 1968, que coincidiu com o movimento estudantil internacional e com o endurecimento do governo militar no Brasil.
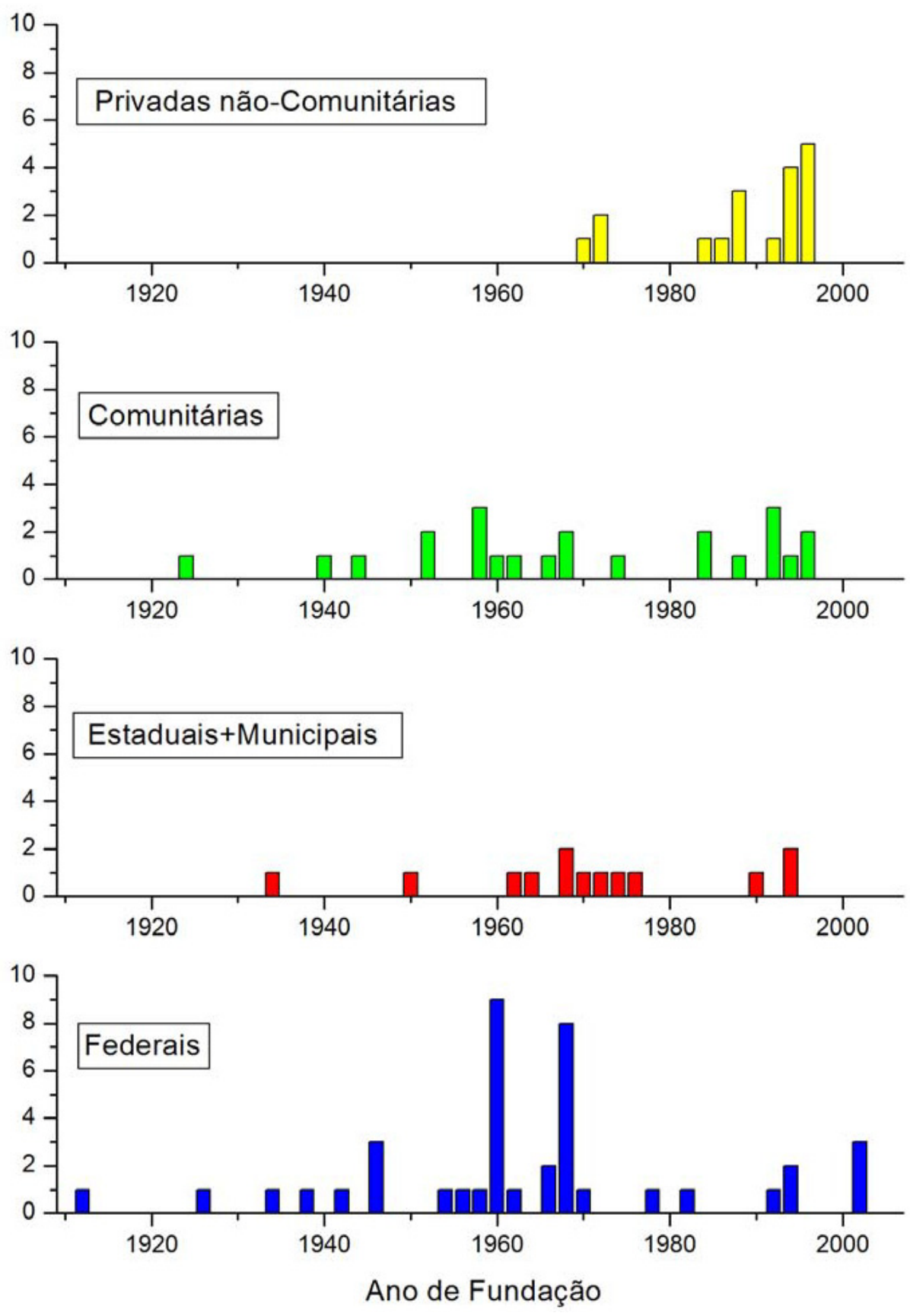

Figura l - Ano de fundação das universidades de pós-graduação no Brasil

Após este segundo surto, esgotou-se a capacidade do governo federal de criar mais universidades e algumas universidades empresariais começaram a sur- 
gir nos primeiros anos da década de 1970. Até o final do governo militar, este quadro permaneceu inalterado. Em 1985, com a redemocratização, as universidades privadas começaram a surgir com maior intensidade.

Tabela 3 - Número de instituições de pós-graduação por região (2003)

\begin{tabular}{l|c|c|c|c|c}
\hline & Fed & $\begin{array}{c}\text { Estad } \\
\text { +Mun }\end{array}$ & CCF & Part & Total \\
\hline Sudoeste & 21 & 10 & 20 & 12 & 63 \\
\hline Sul & 7 & 6 & 12 & 1 & 26 \\
\hline Nordeste & 11 & 2 & 1 & 1 & 15 \\
\hline Centro-Oeste & 4 & 0 & 3 & 1 & 8 \\
\hline Norte & 5 & 0 & 0 & 0 & 5 \\
\hline Total & 48 & 18 & 36 & 15 & 117 \\
\hline
\end{tabular}

A distribuição geográfica da pós-graduação brasileira é bastante irregular. É possível distinguir três macrorregiões com características e IDH (Índice de Desenvolvimento Humano) muito distintos:

- O Sul-Sudeste, com IDH de 0,79 a 0,82, sai na frente com 89 instituições. A população destas duas regiões é de 101 milhões de habitantes, $56 \%$ da população brasileira.

- O Nordeste, com IDH de 0,65 a 0,70, apresenta quinze instituições. A população desta região é de 68 milhões de habitantes, $38 \%$ da população brasileira.

- A Amazônia, com IDH entre 0,70 a 0,72, tem apenas cinco instituições. A população desta região é de cerca de vinte milhões de habitantes.

As distintas macrorregiões são claramente discerníveis na Figura 2, na qual se vê onde estão as pessoas, onde elas geram luz e onde elas geram conhecimento. Na parte superior se vê uma composição fotográfica noturna obtida por satélite ( National Geographic), na qual estão indicadas, em branco, as luzes das cidades (para completar a interpretação da imagem, informamos que em amarelo estão indicados os fogos das queimadas de florestas; em vermelho, a queima de gás natural em poços de petróleo e em azul, a pesca noturna). Na parte inferior temos um mapa demográfico do IBGE (ano 2000) e, superposta, a distribuição das universidades de pesquisa e doutorado, para as quais a área do círculo é proporcional ao número de doutores formados por ano. Fica nítido que na região Sudeste / Sul a maior densidade populacional gera uma maior concentração de luz noturna e, também, conhecimento. Já no Nordeste, as maiores concentrações populacionais geram luz noturna, mas não conhecimento na mesma proporção. Já na Amazônia, mesmo as poucas cidades presentes nos dois mapas geram pouquíssimo conhecimento. 


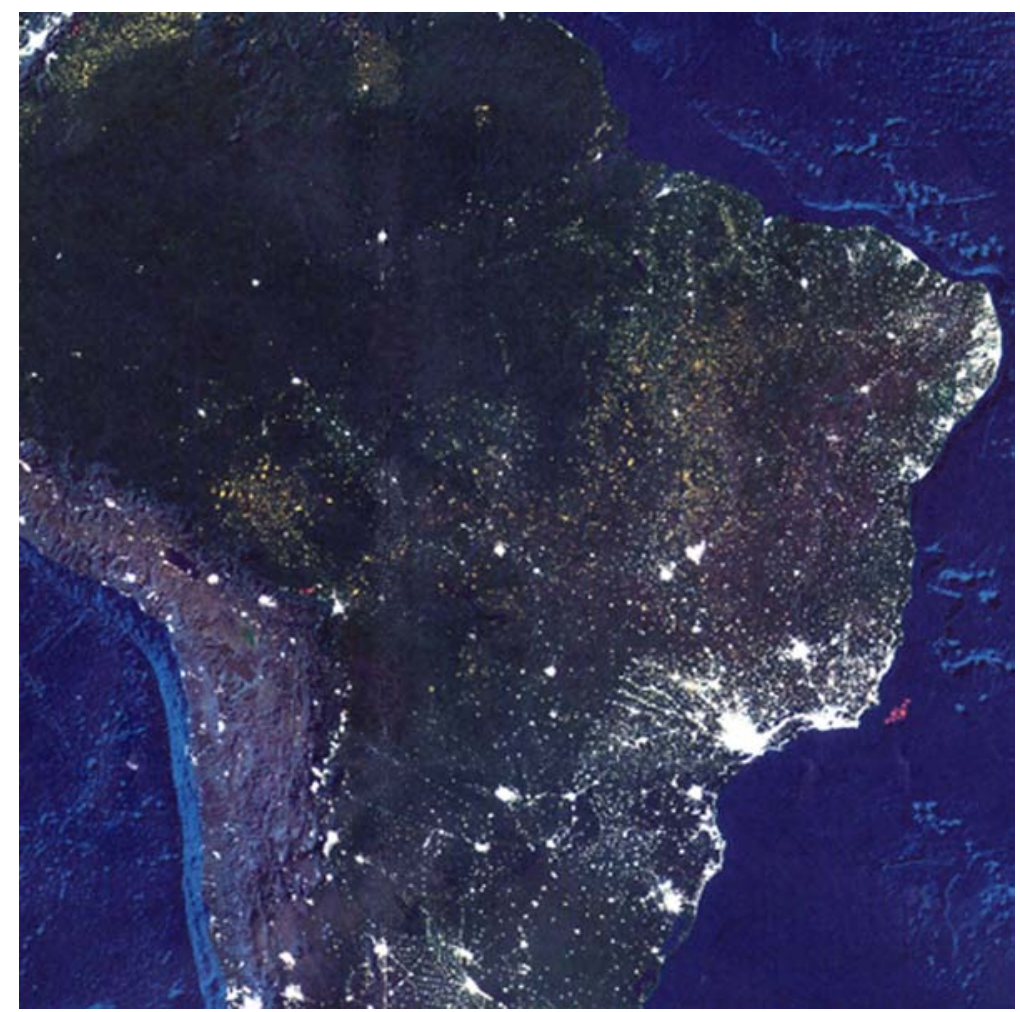

Figura 2 - Onde as pessoas geram luz e onde elas geram conhecimento. A área dos círculos é proporcional ao número anual de concluintes de doutorado.

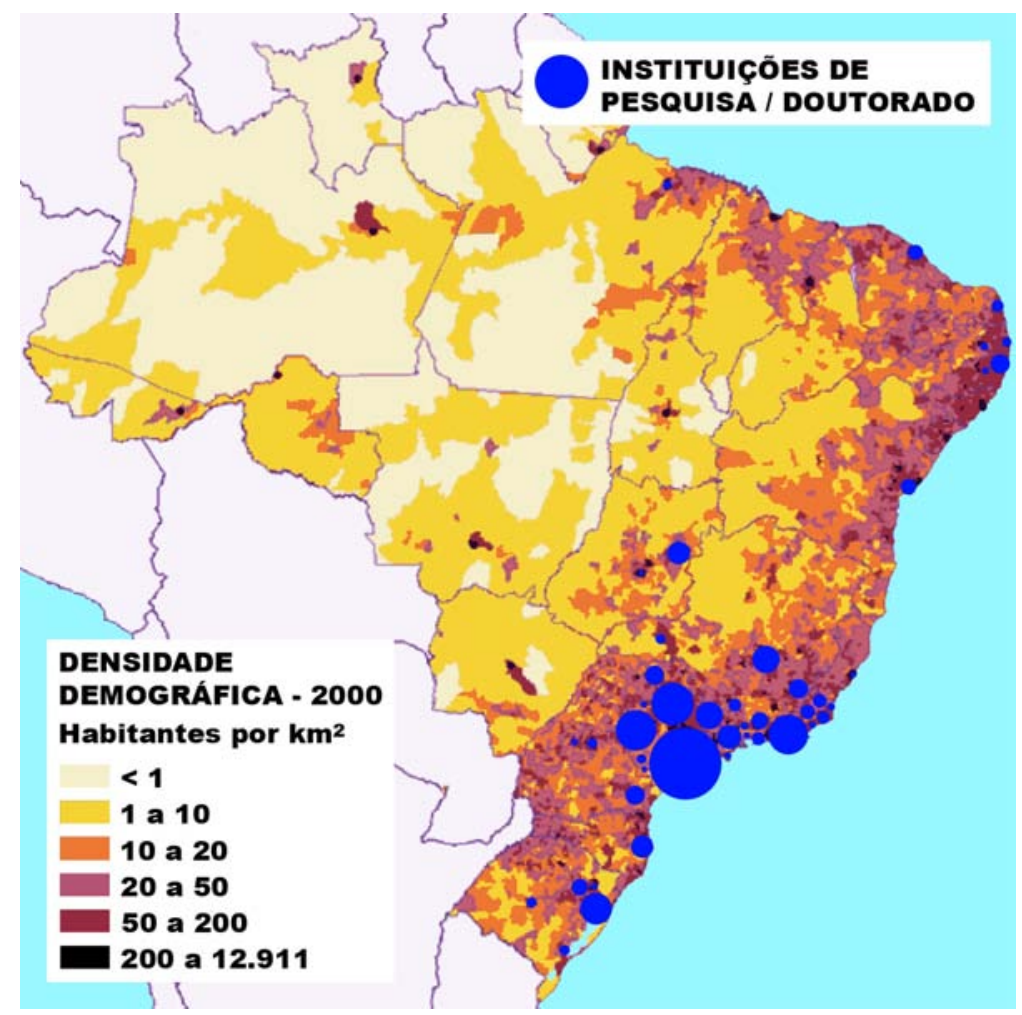

Fonte: IBGE, 2000 
Regiões distintas do País têm distintos padrões de instituições envolvidas na pós-graduação (ver Tabela 3). Na região Sudeste, por exemplo, responsável por mais da metade das 117 instituições de pós-graduação no Brasil, existem 21 instituições federais, dez estaduais ou municipais, vinte comunitárias/ filantrópicas (das quais nove têm origem confessional) e doze particulares. Já a região sul se distingue por ter uma forte presença de instituições comunitárias (doze) e apenas uma particular. O Norte/ Nordeste caracteriza-se pela quase ausência de um sistema comunitário. Temos aqui, talvez, o gargalo mais dramático para a expansão da pós-graduação no País. Com as dificuldades crescentes do sistema federal de se expandir, a ausência de um sistema comunitário abrirá espaço para o setor empresarial, o que, dadas as condições financeiras gerais da população norte-nordestina, dificilmente ocorrerá, ou só será bem-sucedido de forma localizada. Uma alternativa é a expansão via sistema estadual. Alguns estados já experimentam um sistema estadual incipiente, porém promissor, como a Bahia e o Ceará. As experiências do Sul e de São Paulo, no entanto, sugerem que as universidades comunitárias são um segmento significativo e valioso que o Norte/ Nordeste não poderiam dispensar.

Dentro de determinada região também ocorrem situações bastante díspares. No Estado de São Paulo, por exemplo, o sistema universitário é dominado pelas três grandes universidades estaduais. No setor privado, há forte presença das universidades confessionais, num total de cinco. Nota-se, no entanto, um déficit de universidades federais, bem como de um sistema comunitário não-confessional, o que acabou gerando o grande crescimento das universidades particulares. Esta ausência das universidades comunitárias também é notada em Minas Gerais, que possui uma presença forte de universidades federais. A natureza institucional da pós-graduação de Minas Gerais é bastante distinta da de São Paulo e se assemelha à do Nordeste.

É de se notar que existem diversas instituições não universitárias que oferecem programas de pós-graduação. Por exemplo, entre as instituições de pesquisa e de doutorado, notamos a presença da Fiocruz no Rio de Janeiro, Inpe, ITA, FGV-SP e FCMSCSP em São Paulo e Inpa no Amazonas.

\section{Uma comparação estatística entre o Brasil e os Estados Unidos}

Atualmente, o sistema de educação superior dos Estados Unidos é um dos mais admirados do mundo. Particularmente, as suas universidades de pesquisa são consideradas um sólido modelo do que uma boa universidade deve ser. $\mathrm{O}$ sistema de pós-graduação dos EUA não é paradigma para o Brasil. Cabe aqui, no entanto, uma comparação de natureza estatística, uma vez que temos, agora, para os dois países, dados que podem ser comparados. Este tipo de benchmark sempre é útil para entender melhor semelhanças e diferenças entre os dois sistemas e, em decorrência, a realidade da nossa própria pós-graduação.

Os EUA contam com 872 universidades que ministram pós-graduação. Essas universidades dividem-se aproximadamente em partes iguais entre universi- 
dades públicas e privadas sem fins lucrativos. Universidades com fins lucrativos representam apenas cerca de $1 \%$ das universidades americanas aqui consideradas. Já no Brasil, temos 117 instituições envolvidas em pós-graduação (nem todas são universidades), das quais 66 são públicas, 36 são comunitárias/ filantrópicas e quinze são privadas. Os EUA possuem aproximadamente dez vezes mais universidades envolvidas do que o Brasil. Esta também é a proporção entre os Produtos Internos Brutos (PIB) dos dois países. Esta proporcionalidade não é apenas uma curiosidade, mas tem significado mais profundo uma vez que, na era da sociedade da informação e do conhecimento, a educação de alto nível está intimamente ligada ao desempenho econômico de um país. No topo da pirâmide, as universidades de pesquisa e doutorado são dominadas pelo sistema de universidades públicas tanto nos EUA (com 166) como no Brasil (com 32). Neste tipo de universidade, a participação de universidades privadas com fins lucrativos é desprezível em ambos os países, contando-se com apenas duas nos EUA e nenhuma no Brasil. As semelhanças param aí. As universidades de mestrado são dominadas pelas universidades particulares sem fins lucrativos nos EUA. Este tipo de universidade, apesar de ter forte presença no Brasil, tem menor impacto do que nos EUA. No entanto, a maior diferença entre os sistemas é a presença significava das instituições particulares no Brasil, quase inexistentes nos EUA. No Brasil, essa presença limita-se às universidades de mestrado, que é um fenômeno recente e de rápido crescimento.

Tabela 4 - Comparação entre as instituições de ensino superior no Brasil e nos EUA

\begin{tabular}{l|l|c|c|c}
\hline & Públicas & $\begin{array}{c}\text { Comunitárias e } \\
\text { Filantrópicas }\end{array}$ & Privadas & Total \\
\hline
\end{tabular}

BRASIL (2003)*

\begin{tabular}{l|c|c|c|c}
\hline Pesquisa/Doutorado & 36 & 9 & 0 & 45 \\
\hline Mestrado & 31 & 27 & 15 & 73 \\
\hline Graduação & 142 & 292 & 1120 & 1554 \\
\hline Total & $\mathbf{1 7 9}$ & $\mathbf{3 2 8}$ & $\mathbf{1 1 3 5}$ & $\mathbf{1 6 6 2}$ \\
\hline
\end{tabular}

EUA (2000)

\begin{tabular}{l|c|c|c|c}
\hline Pesquisa/Doutorado & 166 & 93 & 2 & 261 \\
\hline Mestrado & 272 & 331 & 8 & 611 \\
\hline Graduação & 1183 & 1251 & 607 & 3041 \\
\hline Total & $\mathbf{1 6 2 1}$ & $\mathbf{1 6 7 5}$ & $\mathbf{6 1 7}$ & $\mathbf{3 9 1 3}$ \\
\hline
\end{tabular}

*No Brasil foram computadas todas as instituições que oferecem pós-graduação, não apenas as universidades. 
Já nas instituições de graduação, a diferença torna-se gritante. Ao passo que os EUA possuem oito vezes mais instituições públicas e quatro vezes mais instituições CCF, o Brasil conta com cerca de duas vezes mais instituições particulares do que os EUA.

Se considerarmos o conjunto das universidades dos EUA e do Brasil, das dez instituições que mais formaram doutores no ano de 2003, quatro são brasileiras (USP, Unicamp, Unesp e UFRJ) e seis são norte-americanas (U. CA Berkely, Nova Southeastern U., U. TX Austin, U. WI Madison, U. IL Urbana-Champaign e U. MI). A primeira do ranking é a USP, que em 2003 formou mais doutores (2.180) do que as três primeiras do ranking americano somadas (2.116).

Tabela 5 - As dez universidades com maior número de concluintes de doutorado $(\mathrm{EUA}+$ Brasil, 2003)

\begin{tabular}{l|l}
\hline 1. Universidade de São Paulo (USP) & 2,180 \\
\hline 2. University of California, Berkeley & 767 \\
\hline 3. Universidade de Campinas (Unicamp) & 747 \\
\hline 4. Nova Southeastern University & 675 \\
\hline 5. University of Texas, Austin & 674 \\
\hline 6. Universidade Estadual de São Paulo (Unesp) & 663 \\
\hline 7. University of Wisconsin, Madison & 653 \\
\hline 8. Universidade Federal do Rio de Janeiro (UFRJ) & 643 \\
\hline 9. University of Illinois, Urbana-Champaign & 618 \\
\hline 10. University of Michigan & 615 \\
\hline
\end{tabular}

Adaptado de Brito Cruz, 2005.

Tabela 6 - Número de doutores formados no Brasil e nos EUA

\begin{tabular}{c|c|c|c}
\hline Ano & Brasil & EUA (total) & EUA (cidadãos) \\
\hline 1988 & 915 & 33,500 & 23,290 \\
\hline 1993 & 1,792 & 39,800 & 26,449 \\
\hline 1998 & 3,949 & 42,645 & 28,456 \\
\hline 2003 & 8,094 & 40,710 & 26,413 \\
\hline
\end{tabular}

Uma comparação entre os números relativos e absolutos de doutores formados nas diversas grandes áreas do conhecimento, em 2003, revela algumas surpresas. É comum ouvir que, no Brasil, se formam muitos doutores nas áreas de pesquisa básica e poucos em pesquisa aplicada. Ao contrário do que reza a 
lenda, a porcentagem dos doutores formados em Engenharia no Brasil (13\%) é semelhante à dos EUA (também 13\%). Já na área da ciência básica, a porcentagem é menor no Brasil (13\% em Ciências Biológicas; 11\% Ciências Exatas e da Terra) do que nos EUA (14\% em Ciências Biológicas; 15\% em Ciências Exatas e da Terra). O mais surpreendente, no entanto, é que o número absoluto de doutores formados nas áreas aplicadas das Ciências Agrárias e da Saúde no Brasil ( 1.026 em Ciências Agrárias; 1.549 em Ciências da Saúde) é semelhante ao dos EUA (1.042 em Ciências Agrárias; 1.633 em Ciências da Saúde), o que, em termos relativos, é cerca de cinco vezes maior para o Brasil quando comparado com os EUA.

Tabela 7 - Comparação entre número de doutores formados por área entre Brasil e EUA em 2003

\begin{tabular}{l|c|c|c}
\hline \multicolumn{1}{c|}{ Área } & Brasil & EUA (total) & EUA (cidadãos) \\
\hline Ciências Agrárias & $1,024(13 \%)$ & $1,042(3 \%)$ & $481(2 \%)$ \\
\hline Ciências Biológicas & $1,028(13 \%)$ & $5,694(14 \%)$ & $3,782(14 \%)$ \\
\hline Ciências da Saúde & $1,549(19 \%)$ & $1,633(3 \%)$ & $1,166(4 \%)$ \\
\hline Ciências Exatas e da Terra & $913(11 \%)$ & $5,963(15 \%)$ & $3,143(12 \%)$ \\
\hline Engenharia & $1,023(13 \%)$ & $5,265(13 \%)$ & $1,898(7 \%)$ \\
\hline Ciências Sociais Aplicadas & $736(9 \%)$ & $6,763(17 \%)$ & $4,947(19 \%)$ \\
\hline Humanidades/Educação & $1,821(22 \%)$ & $14,350(35 \%)$ & $10,996(42 \%)$ \\
\hline
\end{tabular}

Quando se olha apenas para os cidadãos americanos entre os que obtiveram o doutorado, os números são ainda mais surpreendentes. Nas áreas aplicadas, o número absoluto é semelhante ou mesmo menor do que no Brasil. Apenas $7 \%$ dos concluintes fizeram Engenharia, $4 \%$ obtiveram o título na área de Ciências da Saúde e 2\% na área de Ciências Agrárias (ver Tabela 7). Parece claro o risco de brain drain nestes campos, que estão entre as áreas mais aplicadas, com potenciais prejuízos para países como o Brasil. Parece clara, também, a necessidade de se estabelecer uma estratégia para enfrentar uma situação como esta.

Outro fator relevante para o futuro próximo, neste contexto, é o papel da pós-graduação chinesa, que evoluiu de forma dramática nos últimos anos. Tendo formado apenas dezenove doutores no ano de 1983 formou, vinte anos depois, em 2003, um total de 18.625, dos quais 6.242 doutores na área de engenharia (Liu, 2005). Isto representa seis vezes o número formado no Brasil e três vezes o de americanos nesta área. Já o número de novas matrículas no doutorado para o ano de 2005 é de aproximadamente 54 mil, dos quais um terço em engenharia. Por esta perspectiva, a China formará cerca de $50 \%$ dos doutores em engenharia do mundo em um futuro previsível. 


\section{Avaliação da pós-graduação no Brasil}

A Capes (Coordenação de Aperfeiçoamento do Pessoal de Nível Superior do Mistério da Educação) realiza avaliações periódicas de todos os programas de pós-graduação do Brasil. Até o ano de 1998, cada programa recebia um conceito (A, B ....E), de acordo com os critérios estabelecidos por cada comitê de área. A partir de 1998, a Capes adotou uma escala que vai de um a sete. Programas com conceitos seis e sete são considerados de nível internacional. Para assegurar a validação desta métrica, a Capes promove avaliações periódicas destes programas com comitês internacionais de alto nível.

A Capes estabelece um conceito para cada programa de mestrado ou doutorado. No presente trabalho, vamos extrapolar a prática da Capes e atribuir um conceito médio para cada instituição. Para medir a qualidade da pós-graduação de cada universidade/ instituto, tomamos a média ponderada desta instituição, Assumimos a nota Capes de cada programa e adotamos o seu número de doutores formados por ano como peso. Para o ano de 2003, a média nacional para o doutorado foi de cinco e para o mestrado, 4,2.

Vemos que a média ponderada geral brasileira da nota Capes (ver Tabela 10) oscila em torno de cinco (entre 4,9 e 5,1), apesar de variar de forma bem mais significativa de uma grande área para outra. A média da área de Ciências da Saúde, por exemplo, é de 4,2, ao passo que as Ciências Exatas e da Terra têm média de 5,7. Algumas universidades se mantêm sistematicamente abaixo desta média, enquanto outras se mantêm sistematicamente acima; outras, ainda, evoluíram positivamente no período estudado.

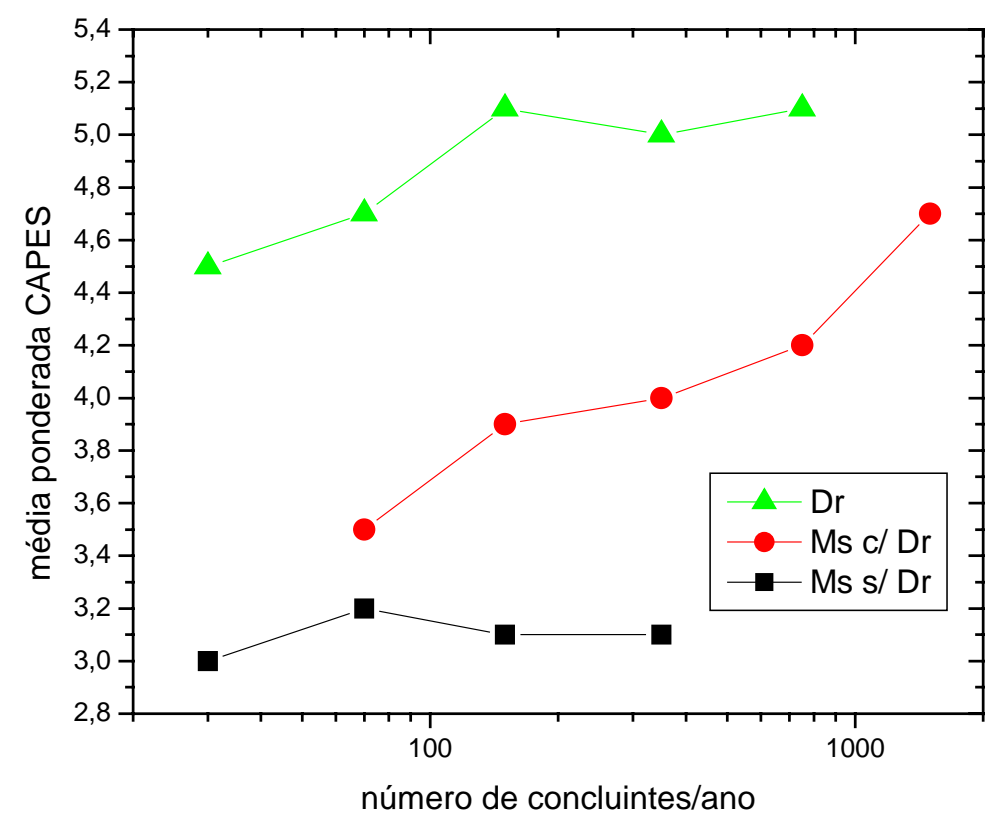

Figura 3 - Médias ponderadas Capes em função do número de concluintes para universidades com ou sem programas de doutorado 
Tabela 8 - Média ponderada da nota da Capes das grandes áreas do conhecimento, por instituição (para 2003). Estão tabuladas apenas as grandes áreas que tiveram vinte ou mais egressos no doutorado. Em negrito estão as áreas que formaram cinqüenta ou mais doutores. Esta tabela indica o mapa da quantidade e da qualidade na pós-graduação brasileira.

\begin{tabular}{|c|c|c|c|c|c|c|c|c|c|c|c|}
\hline Nome & média & $\mathrm{nr} / \mathrm{Dr}$ & $\mathrm{nr} / \mathrm{Pr}$ & AGR & BIO & SAU & $E \& T$ & HUM & CSA & ENG & LLA \\
\hline BRASIL & 5.0 & & 908 & 5.0 & 5.0 & 4.2 & 5.7 & 5.1 & 4.9 & 5.3 & 5.1 \\
\hline USP & 4.9 & 2180 & 200 & 5.4 & 5.3 & 4.2 & 6.0 & 5.4 & 4.6 & 5.0 & 5.1 \\
\hline Unicamp & 5.5 & 747 & 52 & 5.5 & 4.9 & 4.3 & 6.6 & 5.5 & 4.4 & 6.2 & 6.0 \\
\hline Unesp & 4.6 & 663 & 65 & 4.7 & 4.2 & 4.0 & 5.1 & 4.8 & * & * & 4.7 \\
\hline UFRJ & 5.1 & 643 & 68 & * & 5.6 & 4.0 & 5.3 & 5.2 & 4.6 & 5.5 & 5.2 \\
\hline UFRGS & 5.1 & 415 & 55 & 5.0 & 5.5 & 4.0 & 5.9 & 4.9 & * & 5.5 & * \\
\hline UFMG & 5.4 & 296 & 44 & 6.0 & 5.6 & 3.9 & 6.0 & 5.0 & 5.8 & 5.2 & 5.6 \\
\hline UFSC & 5.3 & 210 & 25 & * & * & * & 5.8 & 4.6 & * & 5.6 & 5.0 \\
\hline UnB & 4.9 & 177 & 30 & * & 4.7 & * & 5.3 & 5.6 & * & * & * \\
\hline UFPE & 4.8 & 152 & 32 & & 4.0 & 4.0 & 5.6 & 4.7 & 5.0 & * & * \\
\hline PUC-SP & 5.0 & 313 & 15 & & & & & 4.8 & 5.2 & & * \\
\hline UFV & 5.4 & 158 & 16 & 5.5 & & & & & * & & \\
\hline UFPR & 4.6 & 154 & 24 & 4.0 & 4.8 & * & * & * & 5.7 & * & * \\
\hline UFSCar & 5.7 & 152 & 14 & & 4.7 & & 6.7 & * & & 6.3 & \\
\hline PUC/RJ & 5.4 & 126 & 21 & & & & 5.3 & 5.2 & * & 5.8 & * \\
\hline$P U C / R S$ & 4.2 & 113 & 15 & & * & 4.0 & & 4.1 & * & & 5.0 \\
\hline$U F B A$ & 4.7 & 99 & 16 & & * & 5.0 & * & 4.0 & * & & 4.8 \\
\hline$U F C$ & 4.4 & 94 & 14 & * & 4.5 & * & * & * & * & * & \\
\hline UERJ & 4.9 & 73 & 15 & & * & 4.8 & & * & * & & * \\
\hline UFF & 5.7 & 65 & 20 & * & & * & 5.4 & 6.7 & & & * \\
\hline UFRN & 4.9 & 58 & 10 & & & * & * & 5.0 & & * & \\
\hline UFLA & 4.4 & 72 & 12 & 4.4 & & & & & & & \\
\hline UFRRJ & 5.1 & 66 & 6 & 5.3 & * & & * & * & & & \\
\hline UFSM & 5.0 & 46 & 10 & 4.6 & & & * & & & * & \\
\hline UFU & 4.6 & 43 & 6 & & * & & & & & 4.8 & \\
\hline UEM & 4.7 & 40 & 7 & * & 5.0 & & * & & & * & \\
\hline UFPEL & 4.3 & 34 & 7 & 4.2 & & * & & & & & \\
\hline UEL & 4.4 & 20 & 7 & 4.4 & & & & & & & \\
\hline Unifesp & 4.4 & 245 & 37 & & 5.7 & 4.2 & & & & & \\
\hline Fiocruz & 5.1 & 86 & 7 & & 5.5 & 4.3 & & & & & \\
\hline$F G V-S P$ & 4.0 & 36 & 3 & & & & & & 4.0 & & \\
\hline Inpe & 5.2 & 29 & 6 & & & & 5.3 & & & * & \\
\hline Inpa & 4.4 & 20 & 5 & & 4.4 & & & & & & \\
\hline TOTAL Dr & & 8094 & & 1024 & 1028 & 1549 & 913 & 1283 & 736 & 1023 & 415 \\
\hline
\end{tabular}

Nota: AGR - Ciências Agrárias; BIO - Ciências Biológicas; SAU - Ciências da Saúde; E\&T - Ciências Exatas e da Terra; HUM - Ciências Humanas; CSA - Ciências Sociais Aplicadas; ENG - Engenharias; LLA - Lingüística, Letras e Artes. * Indica que a instituição mantém programas mas formou menos de vinte doutores em 2003. 
Nem todas as áreas do conhecimento têm a mesma qualidade, de acordo com o conceito Capes. Listamos, na Tabela 8 , a média ponderada das universidades, por grande área do conhecimento, nas quais foram formados mais de vinte doutores em 2003.

Na Tabela 8 podemos ver, também, que existem seis universidades fortemente vocacionadas, entre as universidades de pesquisa e de doutorado no Brasil. Destas, quatro são de Ciências Agrárias (UFV, UFLA, UFRRJ e UFRPE), uma Humanística (PUC-SP) e uma especializada em Ciências da Saúde (Unifesp). Temos, ainda, três instituições de pesquisa e de doutorado especializadas que não oferecem programas de graduação (Fiocruz, Inpa e Inpe). Além disto existem outras três instituições especializadas que oferecem cursos de graduação nas suas especialidades (ITA, FGV-SP e FCMSCSP). Pelos critérios aqui utilizados, a Unifesp é a única universidade na categoria de instituição de pesquisa e de doutorado especializada.

\section{Varianças e tendências}

É senso comum afirmar que instituições públicas têm ensino bom e que, em instituições privadas, o ensino tem qualidade freqüentemente questionável. No que diz respeito à pós-graduação, esta afirmação pode ser testada. Na tabela abaixo, mostramos as notas médias da Capes para mestrado e doutorado, tanto para universidades públicas como para filantrópicas e privadas. Podemos notar que existe uma correlação com o tipo de instituição. Instituições de pesquisa e doutorado-diversificadas são as mais bem avaliadas, seguidas das intermediárias e as restritas. No mestrado nota-se que as instituições de pesquisa e de doutorado têm médias mais altas do que as instituições de mestrado. Notamos, com certa surpresa, pois não é o que afirma a lenda, que não há uma forte correlação com a natureza institucional. Para um certo tipo de instituição, as médias são basicamente iguais, tanto para as instituições públicas, quanto para as comunitárias/ filantrópicas como as privadas.

Tabela 9 - Médias Capes por categoria de instituição

\begin{tabular}{l|c|c|c|c|c}
\hline Categoria & \multicolumn{2}{|c|}{ Doutorado } & \multicolumn{3}{c}{ Mestrado } \\
\hline & PUB & CCF & PUB & CCF & PART \\
\hline DrDiv & 5.1 & - & 4.6 & - & - \\
\hline DrInt & 4.9 & 4.9 & 4.2 & 4.5 & - \\
\hline DrRest & 4.5 & 4.7 & 3.8 & 4.0 & - \\
\hline DrEsp & 4.9 & 3.5 & 4.7 & 3.5 & - \\
\hline MsDiv & 3.7 & - & 3.3 & 3.1 & - \\
\hline MsInt & - & - & 3.6 & 3.3 & 3.0 \\
\hline MsRes & - & - & 3.0 & 3.0 & 3.0 \\
\hline MsEsp & 3.7 & 6.3 & 3.2 & 6.3 & 3.2 \\
\hline
\end{tabular}


Uma outra forma de olhar para essa questão é avaliar as médias para instituições em função do número de concluintes de mestrado e de doutorado. $\mathrm{Na}$ Figura 3, mostramos as médias de mestrado para instituições com e sem programas de doutorado e também as médias de doutorado, todas em função do número de concluintes. Vemos, aqui, uma tendência notável. Instituições sem programas de doutorado tendem a ser mal avaliadas, independentemente se são públicas, comunitárias ou privadas. Em instituições que mantêm programas de doutorado, as médias dependem fortemente do número de concluintes. Este efeito se nota também para as médias de doutorado. As instituições com mais de cem concluintes têm médias constantes, mas para instituições com menos de cem concluintes, as médias dependem do número de teses defendidas por ano.

O fato de os programas de mestrado não associados a programas de doutorado serem avaliados em grande parte com nota três (como pode ser visto na Figura 3), indica que os critérios de avaliação destas precisa ser revisto, pois descrimina muito mal a sua qualidade. Não parece razoável que elas tenham quase todas a mesma nota; poderíamos perguntar, por outro lado, para que existem notas dois e um nesta escala?

\section{Universidades no mundo: a situação brasileira}

Como se inserem, em termos de avaliação e qualidade, as universidades brasileiras no contexto mundial? Houve, no ano de 2004, duas pesquisas de nível mundial para estabelecer um ranking de universidades. O primeiro estudo foi conduzido pela universidade chinesa de Shanghai Jiao Tong e o segundo, pelo Times Higher Education, inglês.

O levantamento chinês baseou-se nos seguintes critérios (e pesos): número de ex-alunos que receberam o Prêmio Nobel ou a Medalha Fields (10\%); número de professores que receberam estes prêmios (20\%); pesquisadores altamente citados (20\%); artigos publicados nas revistas Nature ou Science (20\%); artigos publicados no Science Citation Index-expanded e Social Science Citation Index (20\%); soma dos indicadores anteriores dividida pelo tamanho do staff $(10 \%)$.

A pesquisa do Times Higher Education baseou-se na revisão por 1,3 mil especialistas de 88 países, cujo peso foi de $50 \%$. Além disso, considerou o cosmopolitismo dos professores (5\%), dos alunos (5\%), razão docente/ aluno (20\%) e citações por docente (20\%).

Ambas sofrem de deficiências claras. Mas ninguém descobriu, até agora, como fazer tal estudo, sem maiores críticas. Uma das principais críticas que se pode fazer a esse tipo de levantamento é que ele é freqüentemente limitado pela perspectiva anglofônica. É sabido que a cientometria leva em conta preferencialmente a produção em língua inglesa. Ora, boa parte da produção científica brasileira é produzida em português (principalmente nas áreas de Ciências Humanas e de Ciências Sociais Aplicadas) e não computada pelas estatísticas internacionais. Atualmente, temos uma boa indicação do volume da produção da pesquisa brasileira pela base de dados do Scielo (Scientific Eletronic Library Online). Esta 
biblioteca virtual conta atualmente (2005) com cerca de 150 revistas brasileiras que contabilizam impressionantes cifras superiores a dois milhões de acessos por mês. Apenas uma fração pequena destas revistas está indexada no ISI. Entre os dez títulos mais acessados do Scielo, nove são publicados em português. Por outro lado, da mesma forma que o banco de dados do ISI é utilizado pelas áreas das ciências básicas para estabelecer parâmetros de avaliação, o Scielo poderia ser útil nos processos de avaliação, por exemplo, na área das humanidades.

Tabela 10 - Evolução da média ponderada do conceito Capes de doutorado das instituições de pesquisa e de doutorado com mais de cinqüenta concluintes em 2003

\begin{tabular}{|c|c|c|c|c|c|c|c|}
\hline \multirow[t]{2}{*}{ Nome } & \multicolumn{6}{|c|}{ Ano } & \multirow{2}{*}{$\begin{array}{c}\text { Titulados } \\
\begin{array}{c}\text { Núm.Dr. } \\
(2003)\end{array}\end{array}$} \\
\hline & 1998 & 1999 & 2000 & 2001 & 2002 & 2003 & \\
\hline Brasil & 5.0 & 4.9 & 4.9 & 5.1 & 5.0 & 5.0 & 8094 \\
\hline USP & 5.1 & 5.0 & 5.0 & 5.1 & 4.9 & 4.9 & 2180 \\
\hline Unicamp & 5.0 & 5.0 & 4.9 & 5.4 & 5.3 & 5.5 & 747 \\
\hline Unesp & 4.4 & 4.4 & 4.3 & 4.6 & 4.5 & 4.6 & 663 \\
\hline UFRJ & 5.1 & 5.1 & 5.1 & 5.2 & 5.2 & 5.1 & 643 \\
\hline UFRGS & 5.0 & 5.0 & 5.1 & 5.3 & 5.2 & 5.1 & 415 \\
\hline PUC-SP & 5.4 & 5.2 & 5.3 & 5.0 & 4.8 & 5.0 & 313 \\
\hline UFMG & 5.7 & 5.5 & 5.3 & 5.6 & 5.7 & 5.4 & 296 \\
\hline Unifesp & 4.6 & 4.5 & 4.6 & 4.7 & 4.8 & 4.4 & 245 \\
\hline UFSC & 5.3 & 5.2 & 5.2 & 5.4 & 5.2 & 5.3 & 210 \\
\hline UnB & 5.0 & 4.7 & 4.9 & 4.8 & 5.2 & 4.9 & 177 \\
\hline UFV & 6.0 & 6.0 & 5.8 & 5.5 & 5.4 & 5.4 & 158 \\
\hline UFPR & 5.0 & 4.5 & 4.6 & 4.6 & 4.5 & 4.6 & 154 \\
\hline UFPE & 4.2 & 5.0 & 4.6 & 4.9 & 4.9 & 4.8 & 152 \\
\hline UFSCar & 5.0 & 5.1 & 5.2 & 5.7 & 5.7 & 5.7 & 152 \\
\hline PUC-RJ & 5.3 & 5.2 & 5.3 & 5.2 & 5.3 & 5.4 & 126 \\
\hline PUC-RS & 4.6 & 4.5 & 4.5 & 4.4 & 4.3 & 4.2 & 113 \\
\hline UFBA & 4.1 & 4.0 & 4.1 & 4.6 & 4.7 & 4.7 & 99 \\
\hline UFC & 4.6 & 4.5 & 4.5 & 4.4 & 4.5 & 4.4 & 94 \\
\hline Fiocruz & 5.6 & 5.2 & 5.1 & 5.2 & 5.1 & 5.1 & 86 \\
\hline UERJ & 4.5 & 4.3 & 4.5 & 5.0 & 4.9 & 4.9 & 73 \\
\hline UFLA & 4.9 & 5.2 & 5.4 & 4.5 & 4.6 & 4.4 & 72 \\
\hline UFRRJ & 5.0 & 5.2 & 5.3 & 5.2 & 5.2 & 5.1 & 66 \\
\hline UFF & 5.6 & 5.1 & 5.3 & 6.0 & 5.8 & 5.7 & 65 \\
\hline UFRN & 3.0 & 3.5 & 3.4 & 4.4 & 4.5 & 4.9 & 58 \\
\hline
\end{tabular}


De qualquer forma, mesmo levando em conta os caveats mencionados e outros não mencionados, a percepção internacional é de que não existem universidades de classe mundial no Brasil. No levantamento chinês, a universidade brasileira mais bem colocada é a USP, que é uma das últimas colocadas entre as duzentas primeiras. Entre as quinhentas melhores estão ainda a Unicamp, UFRJ e a Unesp. Já o levantamento inglês lista as duzentas melhores universidades do mundo e não há nenhuma universidade brasileira entre elas.

No contexto das universidades de pesquisa, fala-se muito hoje de Universidades de Classe Mundial (World Class Universities). Para Schwartzman (2005), uma universidade de classe mundial deve "desenvolver ciência e tecnologia, mas também cultura, formação geral, conhecimento e capacidade de entender o que ocorre no país e no mundo. Ela deve formar uma grande diversidade de profissionais como professores, diplomatas, altos funcionários, políticos, jornalistas, historiadores etc. Ela deve servir de ponte de contato e comunicação entre o país e o mundo e servir de padrão de referência de qualidade para outras instituições." Além disso, "é a única forma inteligente de lidar com a globalização do ensino".

Uma universidade de classe mundial deve ter algumas características relevantes, incluindo (Altbach, 2003; Schwartzman, 2005):

- Excelência em pesquisa, de qualidade reconhecida internacionalmente. Isto requer fundamentalmente excelentes professores, com talento e dedicação para a pesquisa. A instituição precisa, pois, ter capacidade de recrutar e manter tais quadros. Para isso, ela precisa oferecer salários e condições de trabalho adequados e competitivos, entre outras coisas.

- Liberdade de pesquisa, ensino e expressão, além de atmosfera de excitação intelectual. Adequada infra-estrutura e financiamento para o ensino e a pesquisa.

- Governança moderna e ágil, com autonomia e controle sobre os elementos centrais da vida acadêmica, tais como: admissão de estudantes, currículo, critérios para a concessão de títulos e diplomas, seleção, contratação e dispensa de professores, agilidade para processos de compra e contratos etc., além de cosmopolitismo e diversidade cultural.

O Brasil possui universidades de classe mundial? As nossas universidades de pesquisa e doutorado, de acordo com a descrição acima possuem em algum grau todas as características listadas, mas sempre limitadas de uma forma mais ou menos severa.

O País, a exemplo de outros países desenvolvidos ou em desenvolvimento como a China e a Coréia do Sul, deve fazer um esforço no sentido de se equipar com universidades de classe mundial? A questão, do ponto de vista de políticas públicas, não é saber se devemos tê-las; é saber se em termos de custo/benefício este é o melhor caminho para o desenvolvimento econômico e social do Brasil. Boa pesquisa e boas teses são importantes em si; são também importantes pelo 
potencial de retornarem à sociedade mais do que dela retiraram. Para alcançar este objetivo, a qualidade da pesquisa é um requisito, não apenas uma meta.

Se considerarmos as médias ponderadas da Capes, vemos que nenhuma universidade tem média geral seis ou mais, o que, de acordo com os conceitos da Capes, as colocaria em nível internacional, ao menos no que se refere à pósgraduação. Em certas grandes áreas, no entanto, algumas universidades atingiram este nível, como por exemplo, nas Ciências Agrárias (UFMG), nas Ciências Exatas e da Terra (USP, Unicamp, UFMG e UFSCar), Ciências Humanas (UFF), nas Engenharias (Unicamp e UFSCar) e na Lingüística, Letras e Artes (Unicamp).

Como um todo, as universidades diversificadas que apresentam as maiores médias ponderadas são a Unicamp e a UFMG. Seriam essas, candidatas a se tornarem universidades de classe mundial? Parece ser necessário levar, também, em conta o número de programas de doutorado que a instituição mantém. A USP, com seus duzentos programas de doutorado, tem uma abrangência e complexidade muito maior do que outras universidades bem avaliadas. Talvez por esta razão, entre outras, que Schwartzman (2005) tenha considerado que a USP está entre as poucas instituições brasileiras que tem condições intelectuais, materiais e políticas para encarar o desafio de se tornarem reconhecidas como universidades de classe mundial. Outro aspecto que tem impacto sobre a média institucional diz respeito ao peso relativo das áreas mais bem ou mal avaliadas. Na USP, por exemplo, a área das Ciências da Saúde tem um peso grande e ela é, de todas as grandes áreas, a mais mal avaliada no plano nacional. A UFF e a UFSCar, por outro lado, que também têm médias elevadas, não têm pós-graduação nesta grande área, além de terem um número bem mais restrito de programas. Isto ilustra a necessidade de o conceito de média ponderada institucional ser analisado com cautela.

\section{Referências}

ABRUC - Associação Brasileira de Universidades Comunitárias (ver http://www. abruc.org.br)

ALTBACH, Phillip G. "The Costs and Benefits of World-Class Universities". International Higher Education, primavera de 2003.

BRITO CRUZ, C. H. Pesquisa e Universidade, 2005 http://www.usp.br/iea/ensinosuperior/

CAPES/ MEC. Coordenação de Aperfeiçoamento do Pessoal de Nível Superior - Ministério da Educação (ver http://www.capes.gov.br).

Carnegie Foundation for the Advancement of Teaching (http://www.carnegiefoundation.org/Classification/).

DURHAM, Eunice R. "Educação Superior, Pública e Privada (1808-2000)". Em BROCK, C. e SCHWARTZMAN, S. (orgs.). Os desafios da Educação no Brasil. Rio de Janeiro, Nova Fronteira, 2005. 
INEP / MEC - Instituto Nacional de Estudos e Pesquisas Anísio Teixeira, Ministério da Educação (ver http://www.inep.gov.br)

LIU, N. C. "The Differentiation and Classification of Chinese Universities and the Building of World-Class Universities in China". Em ALTBACH, P. et al., no prelo.

LOBO, Roberto L. S. Folha de S. Paulo, 20/9/2004.

MARTINS, C. B. “O ensino superior brasileiro nos anos 90”. Em São Paulo em Perspectiva, 14(1), 2000.

SCIELO - Scientific Electronic Library Online (http://www.scielo.org.br )

SCHWARTZMAN, S. “A Universidade de São Paulo e a questão universitária no Brasil”. Em Os desafios do ensino superior no Brasil. São Paulo, Instituto de Estudos Avançados da USP, 2005.

SHANGHAI JIAO TONG UNIVERSITY. http://www.sjtu.edu.cn/www/english/

STEINER, J. Diferenciação e classificação das instituições de ensino superior no Brasil, 2005 http://www.usp.br/iea/ensinosuperior/

Survey of Earned Doctorates. http://www.norc.uchicago.edu/issues/docdata.htm

TIMES HIGHER EDUCATION. ver http://www.thes.co.uk/

RESUMO - ANALISAMOS a pós-graduação brasileira no que diz respeito à sua diversidade institucional e a sua qualidade. Esta diversidade cresceu nas últimas décadas. O Brasil tinha, em 2003, um total de 45 instituições de pesquisa e doutorado e 73 de mestrado, (além de 1.554 instituições de ensino de graduação). A distribuição geográfica das instituições envolvidas com pós-graduação é bastante irregular.

A relação entre o número de instituições envolvidas na pós-graduação no Brasil e nos EUA mantém a mesma proporção que entre o PIB dos dois países. As universidades de pesquisa e de doutorado brasileiras, assim como as americanas, são basicamente públicas, seguidas de universidades comunitárias que, no caso do Brasil, têm origem majoritariamente confessional. Ao contrário do que normalmente se afirma, o Brasil forma maior porcentagem de doutores em áreas aplicadas como Ciências Agrárias e Ciências da Saúde do que os EUA. Isto implica a existência de um sério risco de brain drain nestas áreas. A qualidade dos cursos de mestrado e de doutorado não depende fortemente da natureza da instituição, se pública, comunitária/ filantrópica ou privada. A qualidade dos cursos de mestrado de uma instituição depende do número de dissertações da instituição mas, também, do fato de ela ter ou não programas de doutorado. Diferentes áreas do conhecimento parecem ter distintos graus de amadurecimento, sendo que a Área de Ciências da Saúde (com 4.2) tem a pior avaliação Capes e a Área de Ciências Exatas e da Terra, a melhor (5.7). Algumas universidades já atingiram o nível internacional em algumas grandes áreas.

Palavras-chave: Pós-graduação; avaliação; diversidade; universidades.

ABSRACT - THE InSTITUTIONS that offer graduate programs in Brazil are analyzed with respect to their diversity and quality. This diversity has grown in the last decades. Brazil had, in 2003, a total of 45 doctoral/research institutions, 73 that offered master degree (besides 1,554 institutions that offered graduate courses). The geographic distribution of these institutions is quite irregular. 
The relation between the number of institutions involved in graduate programs in Brazil and USA is similar to the proportion of the GNP of the two countries. The doctoral/ research institutions in Brazil, like in the USA, are basically public, followed by community-type institutions that, in the case of Brazil, have often faith-relate origin. Contrary to common believes, Brazil graduates a larger percentage of doctorates in applied areas then the USA. In fields like Agrarian Sciences and Health Sciences, the absolute numbers are similar. This implies a clear risk of future brain drain in these fields of knowledge, potentially damaging for countries like Brazil.

The quality of the master and doctorate programs does not depend strongly on the administrative nature, if public, community related or private. The quality of the master programs of an institution depends on the number of its graduates but also on the fact that the institution offers or not doctoral programs. Distinct fields of knowledge seem to mature differently. Health Sciences present the worst Capes scores (4.2) while Exact and Earth Sciences present the best (5.7). Some institutions have reached international level in some of the large areas of knowledge.

Key-words: Graduate studies; evaluation; diversity; universities.

João E. Steiner é diretor do Instituto de Estudos Avançados (USP) e professor do Instituto de Astronomia Geofísica e Ciências Atmosféricas (USP). @ - steiner@astro.iag.usp.br

O autor agradece a Dra. Marisa Cassim, a Profa. Eunice Durham e a Ronaldo Uller, pelas sugestões, e a Mauro Bellesa, Sandra Sedini e Renato M. Steiner pela assistência técnica. Texto recebido em 10/6/2005 e aceito em 8/7/2005. 


\section{Apêndice}

\section{Definições}

A classificação proposta está baseada em três dimensões: o grau mais elevado de diplomação; a diversidade acadêmica e o tamanho; a natureza administrativa. A seguir, listamos as definições de cada categoria proposta por Steiner (2005). Esta classificação é uma adaptação do sistema Carnegie à situação Brasil.

- Instituições de pesquisa e de doutorado: oferecem tipicamente uma ampla gama de programas de bacharelado e estão comprometidas com o ensino de pós-graduação até o doutorado.

* Instituições de Pesquisa e de Doutorado Diversificadas (DrDiv), que oferecem pelo menos 25 programas de doutorado em pelo menos seis grandes áreas do conhecimento e formaram pelo menos 150 doutores / ano no período considerado.

* Instituições de Pesquisa e de Doutorado Intermediárias (DrInt), que oferecem pelo menos dez programas de doutorado em pelo menos duas grandes áreas do conhecimento e formaram pelo menos cinqüenta doutores/ ano no período considerado.

* Instituições de Pesquisa e de Doutorado Restritas (DrRes), que formaram pelo menos dez doutores/ ano em pelo menos três programas ou formaram pelo menos vinte doutores/ ano no total.

* Instituições de Pesquisa e Doutorado Especializadas (DrEsp), que oferecem programas de doutorado, mas oferecem diplomas de graduação em uma única área do conhecimento ou não oferecem cursos de graduação e formaram pelo menos vinte doutores/ ano no período considerado.

- Instituições de mestrado: oferecem tipicamente uma ampla gama de programas de bacharelado e estão comprometidas com o ensino de pós-graduação até o mestrado.

* Instituições de Mestrado Diversificadas (MsDiv), que oferecem pelo menos cinco programas de mestrado e formaram pelo menos 75 mestres/ ano no período considerado.

* Instituições de Mestrado Intermediárias (MsInt), que oferecem pelo menos três programas de mestrado e formaram pelo menos quarenta mestres / ano no período considerado.

* Instituições de Mestrado Restritas (MsRes), que formaram pelo menos vinte mestres/ ano no período considerado.

* Instituições de Mestrado Especializadas (MsEsp), que oferecem programas de mestrado, mas oferecem diplomas em um único campo do conhecimento. Formaram pelo menos vinte mestres / ano no período considerado.

- Instituições de graduação: oferecem ensino de graduação.

* Instituições de Graduação Diversificadas (GrDiv), com mais de mil concluintes por ano em pelo menos 25 cursos.

* Instituições de Graduação Intermediárias (GrInt), com um número anual mínimo de concluintes de quinhentos em pelo menos dez cursos.

* Instituições de Graduação Restritas (GrRes), com menos de quinhentos concluintes por ano. 
* Instituições de Graduação Especializadas (GrEsp), que oferecem diploma em um único campo do conhecimento:

»Tecnológicas

» Licenciatura (formação de professores)

» Profissionais Liberais

»Belas Artes

Além disso, as instituições são divididas de acordo com os critérios do Inep em três categorias administrativas:

- públicas (federais, estaduais e municipais);

- CCF (comunitárias, confessionais e filantrópicas); e

- particulares (de caráter empresarial, não comunitário).

Observação: Os dados primários para a pós-graduação foram obtidos do site da Capes. A classificação administrativa foi obtida do banco de dados do Inep.

* Indica que a instituição é membro da Abruc - Associação Brasileira das Universidades Comunitárias.

Uma listagem das instituições classificadas, com o respectivo número de cursos e concluintes, pode ser encontrado em Steiner (2005).

\section{A - Instituições de pesquisa e doutorado (2003)}

AI - Universidades de Pesquisa e Doutorado - Diversificadas (DrDiv)

Públicas: USP - Universidade de São Paulo. Unicamp - Universidade Estadual de Campinas. Unesp - Universidade Estadual Paulista Júlio de Mesquita Filho. UFRJ Universidade Federal Rio de Janeiro. UFRGS - Universidade Federal do Rio Grande do Sul. UFMG - Universidade Federal de Minas Gerais. UFSC - Universidade Federal de Santa Catarina. UnB - Universidade de Brasília. UFPE - Universidade Federal de Pernambuco.

\section{A2 - Universidades de Pesquisa e Doutorado - Intermediárias (DrInt)}

Públicas: UFV - Universidade Federal de Viçosa. UFPR - Universidade Federal do Paraná. UFSCar - Universidade Federal de São Carlos. UFBA - Universidade Federal da Bahia. UFC - Universidade Federal do Ceará. UERJ - Universidade do Estado do Rio de Janeiro. UFF - Universidade Federal Fluminense. UFRN - Universidade Federal do Rio Grande do Norte.

CCF: *PUC-SP - Pontifícia Universidade Católica de São Paulo. *PUC-RJ Pontifícia Universidade Católica do Rio de Janeiro. *PUC-RS - Pontifícia Universidade Católica do Rio Grande do Sul.

\section{A3 - Universidades de Pesquisa e Doutorado - Restritas (DrRes)}

Públicas: UFLA - Universidade Federal de Lavras. UFRRJ - Universidade Federal Rural do Rio de Janeiro. UFSM - Universidade Federal de Santa Maria. UFU - 
Universidade Federal de Uberlândia. UEM - Universidade Estadual de Maringá. UFPB - Universidade Federal da Paraíba. UFPEL - Universidade Federal de Pelotas. UFPA - Universidade Federal do Pará. UENF - Universidade Estadual do Norte Fluminense. UFCG - Universidade Federal de Campina Grande. UFRPE - Universidade Federal Rural de Pernambuco. UEL - Universidade Estadual de Londrina. UFG - Universidade Federal de Goiás. UFES - Universidade Federal do Espírito Santo.

CCF: *Puccamp - Pontifícia Universidade Católica de Campinas. *Unisinos Universidade do Vale do Rio dos Sinos. ${ }^{*}$ Umesp - Universidade Metodista de São Paulo. UGF - Universidade Gama Filho.

\section{A4 - Instituições de Pesquisa e Doutorado - Especializadas (DrEsp)}

Públicas: Unifesp - Universidade Federal de São Paulo. Fiocruz - Fundação Instituto Osvaldo Cruz. Inpe - Instituto Nacional de Pesquisas Espaciais. Inpa - Instituto Nacional de Pesquisas da Amazônia. ITA - Instituto Tecnológico de Aeronáutica .

CCF: FGV/SP - Fundação Getúlio Vargas-SP. FCMSCSP - Faculdade de Ciências Médicas da Santa Casa de São Paulo.

\section{B - Instituições de mestrado (2003)}

\section{BI - Universidades de Mestrado - Diversificadas (MsDiv)}

Públicas: UFAM - Universidade Federal do Amazonas. FUFMS - Fundação Universidade Federal do Mato Grosso do Sul. UFMT - Universidade Federal do Mato Grosso. FUFSE - Fundação Universidade Federal de Sergipe. UFAL - Universidade Federal de Alagoas. Funece - Fundação Universidade Estadual do Ceará. UFOP - Universidade Federal de Ouro Preto. FURG - Fundação Universidade do Rio Grande.

CCF: *UPM - Universidade Presbiteriana Mackenzie. *PUC/MG - Pontifícia Universidade Católica de Minas Gerais. ${ }^{\star}$ Univali - Universidade do Vale do Itajaí. *PUC/PR - Pontifícia Universidade Católica do Paraná. *UCB - Universidade Católica de Brasília. Unifor - Universidade de Fortaleza. Ulbra - Universidade Luterana do Brasil.

\section{B2 - Instituições de Mestrado - Intermediárias (MsInt)}

Públicas: CEFET/PR - Centro Federal de Educação Tecnológica do Paraná. UFJF - Universidade Federal de Juiz de Fora. FURB - Fundação Universidade Regional de Blumenau. UFMA - Universidade Federal do Maranhão. Unifei - Universidade Federal de Itajubá. Unirio - Universidade do Rio de Janeiro.

CCF: *Unimep - Universidade Metodista de Piracicaba. *UCDB - Universidade Católica Dom Bosco. *UCGO - Universidade Católica de Goiás. *Univap Universidade do Vale do Paraíba. ${ }^{\star} \mathrm{UPF}$ - Universidade de Passo Fundo. ${ }^{*}$ USF Universidade São Francisco. *Unijuí - Universidade Regional do Nordeste do Estado do Rio Grande do Sul. Unesa - Universidade Estácio de Sá.

Particulares: Unifran - Universidade de Franca. UTP - Universidade Tuiutí do Paraná. Unicid - Universidade Cidade de São Paulo. Unip - Universidade Paulista. Unifacs - Universidade de Salvador.

\section{B3 - Instituições de Mestrado - Restritas (MsRes)}

Públicas: Unitau - Universidade de Taubaté. UDESC - Universidade do Estado 
de Santa Catarina. FUPI - Fundação Universidade Federal do Piauí. UESC - Universidade Estadual de Santa Cruz. Unioeste - Universidade Estadual do Oeste do Paraná. UNIR - Universidade Federal de Rondônia.

CCF: Unaerp - Universidade de Ribeirão Preto. * UCP - Universidade Católica de Petrópolis. *UNISC - Universidade de Santa Cruz do Sul. *UCPEL - Universidade Católica de Pelotas. *UCS - Universidade de Caxias do Sul. Unisa - Universidade de Santo Amaro. Unoeste - Universidade do Oeste Paulista. UCB - Universidade Castelo Branco. *Univille - Universidade da Região de Joinville.

Particulares: Unimarco - Universidade São Marcos. Uninove - Centro Universitário Nove de Julho. UMC - Universidade de Mogi das Cruzes. Unimes - Universidade Metropolitana de Santos. Uniderp - Universidade para o Desenvolvimento do Estado e da Região do Pantanal.

B4 - Instituições de Mestrado - Especializadas (MsInt)

Públicas: IME - Instituto Militar de Exército. IPT - Instituto de Pesquisas Tecnológicas. IRBr - Instituto Rio Branco. IAC - Instituto Agronômico de Campinas. Fundinop - Faculdade Estadual de Direito do Norte Pioneiro. Ence - Escola Nacional de Ciências Estatísticas. FJP - Fundação João Pinheiro. CEFET/MG Centro Federal de Educação Tecnológica de Minas Gerais. UFRA - Universidade Federal Rural da Amazônia. Ipen - Instituto de Pesquisas Energéticas e Nucleares. Faenquil - Faculdade de Engenharia Química de Lorena.

CCF: FGV/RJ - Fundação Getúlio Vargas-RJ. IMPA/OS - Instituto de Matemática Pura e Aplicada - Organização Social. EST - Escola Superior de Teologia.

Particulares: IBMEC - Faculdade de Economia e Finanças do Instituto Brasileiro de Mercado de Capitais. SLMANDIC - Centro de Pesquisas Odontológicas São Leopoldo Mandic. Facesp - Faculdade de Ciências Econômicas de São Paulo. ITE Instituto Toledo de Ensino. SBI - Sociedade Brasileira de Instrução. 